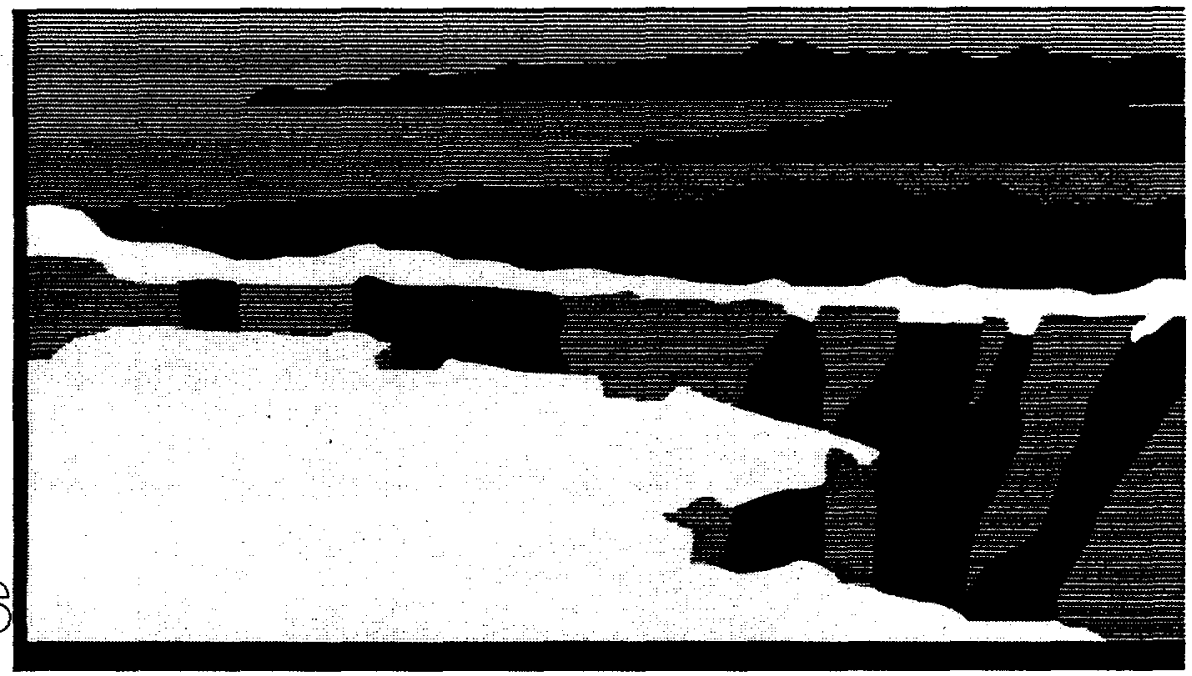

Los Alamos National Laboratory, an affirmative actioniequal opportunity employer, is operated by the University of California for the U.S. Department of Energy under contract W-7405-ENG-36. By acceptance of this article, the publisher recognizes that the U.S. Government retains a nonexclusive, royalty-free license to publish or reproduce the published form of this contribution, or to allow others to do so, for U.S. Govemment purposes. The Los Alamos National Laboratory requests that the publisher identify this article as work performed under the auspices of the U.S. Department of Energy. 


\section{DISCLAIMER}

This report was prepared as an account of work sponsored by an agency of the United States Government. Neither the United States Government nor any agency thereof, nor any of their employees, make any warranty, express or implied, or assumes any legal liability or responsibility for the accuracy, completeness, or usefulness of any information, apparatus, product, or process disclosed, or represents that its use would not infringe privately owned rights. Reference herein to any specific commercial product, process, or service by trade name, trademark, manufacturer, or otherwise does not necessarily constitute or imply its endorsement, recommendation, or favoring by the United States Government or any agency thereof. The views and opinions of authors expressed herein do not necessarily state or reflect those of the United States Government or any agency thereof. 


\section{DISCLAIMER}

Portions of this document may be illegible in electronic image products. Images are produced from the best available original document. 


\title{
ENVELOPE OSCILLATIONS AND HALO FORMATION IN BUNCHED BEAMS: A PERTURBATIONAL APPROACH*
}

\author{
CHRISTOPHER K. ALLEN \\ MS H817, Los Alamos National Laboratory \\ Los Alamos, New Mexico 87545 \\ E-mail: ckallen@lanl.gov
}

\begin{abstract}
We examine in detail the first-order vibration modes of a bunched beam then apply the resultsto the analysis of halo formation using the particle-core model. The three-dimensional envelope equations are derived from first principle in order to avoid some discrepancies currently found in the literature. From the full envelope equations, we employ perturbation analysis to compute analytic expressions for the natural oscillation frequencies and corresponding oscillation modes of a beam bunch. Moreover, the mode excitations due to a periodic accelerator structure can be estimated. We then use the results of the envelope analysis as a starting point in the study of halo formation in bunched beams. An overview of the current nonlinear analysis using the particle-core model is presented. The collected results of this study are to be published at a later time.
\end{abstract}

\section{Introduction}

The aim of this article is to carefully analyze and document the oscillatory behavior of bunched beams in periodic accelerator structures. From the particle-core model, it is known that bunch oscillations may induce halo growth in beams. The primary culprit seems to be the natural oscillation modes, or eigenmodes, of the beam core. Therefore, we wish to have a thorough quantitative understanding of these eigenmodes on which to base further investigation into halo growth. This article presents the findings that we believe to be accurate and useful in our endeavor to extend the analysis of the particle-core model. Our final goal is to extract quantitative results on halo growth using nonlinear analysis of the particle-core model.

We begin by developing, in detail, the natural oscillation modes of a bunched beam. There are discrepancies in the literature over the appropriate form of the envelope equations for a bunched beam and, not surprisingly then, there are inconsistencies in the results previously published on the natural modes of a bunch. Thus, we develop the envelope equations for a bunched beam on first principle and the results on core oscillations follow directly from this analysis. As shown previously by Gluckstern et al [6], this approach is necessary to obtain correct results from the perturbation analysis.

\footnotetext{
- Work supported by the U.S. Department of Energy
} 


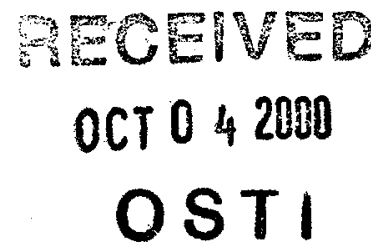

As mentioned, it is the ultimate goal of this work to generate some quantitative results from the particle-core model. Still, much of the quantitative results on halo growth are achieved through numeric experiments and simulation (for example, the "peanut" diagram of stable orbits). Since halo formation in the particle-core model is nonlinear in nature, we must employ nonlinear methods to generate quantitative results. The primary tools used here are a multi-scale perturbational approach [8][10] used in conjunction with the Fredholm alternative theorem [13]. An outline of our current work in progress is presented in the final section.

\subsection{The Particle-Core Beam Model}

Recently we have seen much research activity into the phenomena of halo formation in charged particle beams [2][6][14][15][16]. This is due, in part, to the success of the particle-core beam model used in these investigations. There an independent beam "core" generates the collective fields of the beam. The motion of a test particle is then followed under the action of the fields of the core and those of the external focusing system. (The core behavior is considered independent because the fields of the test particles do not interact.) From this model, it has been discovered that there are potential parametric resonances between oscillations of the beam core and the motion of individual beam particles. In essence, particles within the core may experience parametric "kicks" which eventually drive them outside the core. This mechanism is primarily nonlinear, a particle's oscillation frequency depends upon its amplitude. In this manner, the coupling between core and particle breaks once the amplitude is large enough to pull the particle from resonance.

Within the beam core, individual particles undergo "betatron oscillations" in response to the external focusing system. This is typically the case even if the core distribution is stationary (e.g., a finite beam emittance). Additionally, the core may also experience collective oscillations in an accelerator system. In an alternating gradient (AG) focusing system, for example, the core is designed to oscillate synchronously with the focusing structures. The beam core also has "natural" oscillation modes, or resonances, which can be inadvertently excited in accelerator systems (e.g., from a mismatch). The particle-core model has shown that beam core oscillations are a major cause of halo growth.

The particle-core model has provided us with a qualitative understanding of halo formation. Using a simple linear model for the internal fields of the oscillating core, the individual particle equations of motion then have the form of Hill's equation. Hill's equation has been studied extensively and, although it is linear, it is known to be unstable for particular parameter values. In accelerator parlance, this means that when the betatron frequencies of the particles become close to that of the beam core oscillation frequency (or a suitable harmonic), parametric resonance ensues and the particle becomes unstable. The linear model suggests that the particle's amplitude would then begin increasing without bound. However, once

CKAllen_ICFA_Workshop.doc submitted to World Scientific 02/10/00: $2 / 20$ 
the particle has been kicked outside the core, the collective fields are no longer linear, falling off rapidly. The particle "feels" the fields of the focusing system disproportionally more than those of the core, its betatron frequency do then change and the resonance is broken.

In order to avoid instabilities associated with core oscillations from AG focusing; it is widely known that the betatron oscillations must be kept significantly less than that of the focusing period. Specifically, the particle's phase advance per accelerator period must be less than 90 degrees. Thus, the resonances from the periodic machine structure can be avoided with proper design. The natural modes of the beam core present a different problem, however. Typically, the frequencies of these oscillations are much less than that of the underlying accelerator structure. Herein usually lies the threat of parametric resonance.

\subsection{Halo Formation}

The analysis is assumed to take place in the beam frame where motion is nonrelativistic and magnetic interactions are negligible. Currently, two approaches are being employed, they differ in the manner in which the self electric fields are expanded. Each avenue has its own merits. In the first approach, the electric fields are expanded directly in terms of the perturbation parameter. This procedure has the benefit of simplicity, yet the resulting expansions tend to be rather complicated algebraic expressions. In the second approach, we first approximate the self-fields with a polynomial expansion in the coordinates $x, y$, and $z$. The expansion is constructed so that the resultant approximate fields represent the true fields in an averaged sense (i.e., a Lesbesgue sense), rather than simply Taylor expanding. The coefficients of the monomials are functions of the bunch envelopes and, consequently, vary in time in accordance with any beam core oscillations. If we consider only the linear monomial of the field expansion, and consider only firstorder variations of the coefficients, then Hill's equation is the consequence. That is, we recover the linear analysis seen previously in the literature. This approach has the advantage that we can see directly the effects of the various linear and nonlinear components of the self-fields.

Once the self-field expressions are expanded in the perturbation parameter, the analysis proceeds identically. The equations of motion for individual particles are formed, the various orders are collected then examined. As discussed above, the perturbation analysis also employs a multi-scale expansion in the dependent variable so that we may eliminate secular terms. The Fredholm alternative theorem provides us with the formal mechanism to do this, typically furnishing the dispersion relation between frequency and amplitude of the test particles. The major difference in the two approaches after this point is that the former requires information on the actual charge distribution whereas the later does not (the distribution information is "averaged out" during the expansion of the self-fields).

CKAllen_ICFA_Workshop.doc submitted to World Scientific 02/10/00: $3 / 20$ 
The current work on halo formation is still very much a work in progress. Thus, we present the techniques employed and preliminary findings without any definite conclusions. At the very least, we expect to gain further qualitative insight into the formation of halo and identify critical coupling conditions between core and particle motions. What we hope for in the continued analysis is quantitative conclusions on halo phenomena such as halo amplitude, time constants for the halo formation, and perhaps the percentage of beam constituting the halo. These findings will be published later.

\section{The Bunched Beam Envelope Equations}

We model the bunch using the equations for the equivalent uniform beam. These equations follow directly from the rms envelope equations. Technically speaking, the rms envelope equations for a bunched beam are dependent upon the charge density. However, Sacherer showed that this dependence is quite weak [12]. . The implication is that the second-order spatial moments (i.e., the rms envelopes) of any bunched beam behave the same, irrespective of the particular particle distribution. Consequently, we are justified in modeling any laboratory beam with any beam having the same second moments $\left\langle x^{2}\right\rangle,\left\langle y^{2}\right\rangle$ and $\left\langle z^{2}\right\rangle$. Since the uniform beam has well-defined boundaries, this distribution is typically preferred.

\subsection{Exact Form of the Envelope Equations}

Let $s$ denote the path length along the design trajectory, our independent variable. Denoting the bunch envelopes (semi-axes) as $X(s), Y(s)$ and $Z(s)$ for the $x, y$ and $z$ planes, respectively, the envelope equations for the equivalent uniform beam are [1]

$$
\begin{array}{r}
X^{\prime \prime}+k_{x}^{2}(s) X-K \frac{10 \pi \varepsilon_{0}}{q N^{2}} \frac{\left\langle x E_{x}\right\rangle}{X}-\frac{\varepsilon_{x}^{2}}{X^{3}}=0, \\
(1) Y^{\prime \prime}+k_{y}^{2}(s) Y-K \frac{10 \pi \varepsilon_{0}}{q N^{2}} \frac{\left\langle y E_{y}\right\rangle}{Y}-\frac{\varepsilon_{y}^{2}}{Y^{3}}=0, \\
Z^{\prime \prime}+k_{z}^{2}(s) Z-\gamma^{2} K \frac{10 \pi \varepsilon_{0}}{q N^{2}} \frac{\left\langle z E_{z}\right\rangle}{Z}-\frac{\varepsilon_{z}^{2}}{Z^{3}}=0,
\end{array}
$$

where the quantity $\gamma$ is the relativistic factor, $K$ is the generalized beam perveance, $N$ is the number of bunch particles and the $k_{l}^{2}(s)$ represent the focusing strengths in the respective planes $i \in\{x, y, z\}$. The emittances $\varepsilon_{x}, \varepsilon_{y}$ and $\varepsilon_{z}$ are the effective emittances for the uniform beam, given by five times the rms values. Thus, under our assumptions we are left with the unknowns $\left\langle x E_{x}\right\rangle,\left\langle y E_{y}\right\rangle$, and $\left\langle z E_{z}\right\rangle$, which are the weighted moments of the self-electric field components. Other than the 
emittances, these are the only quantities that depend explicitly on the actual bunch distribution.

\subsection{Computation of Electric Field Moments}

It is possible to compute the field moments explicitly (in terms of elliptic integrals) for bunches having ellipsoidal symmetry in configuration space. By ellipsoidal symmetry, we imply that the charge distribution $\rho$ has the form

(2) $\rho(x, y, z)=q f\left(\frac{x^{2}}{a^{2}}+\frac{y^{2}}{b^{2}}+\frac{z^{2}}{c^{2}}\right)$.

where $f(\cdot)$ represents the profile of the distribution, $q$ is the unit charge, and $a$, $b, c$ represent the $x, y, z$ semi-axes of the reference ellipsoid, respectively. This

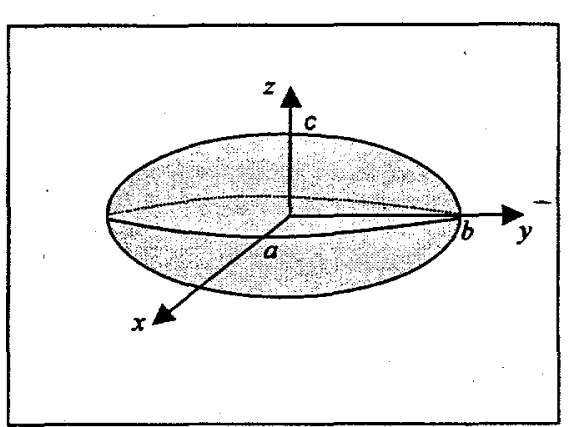

Figure 1: reference ellipsoid of ellipsoidally symmetric charge density situation is depicted in Figure 1.

We start from the following formula for the self-electric potential of such a bunch [7][9]:

(3) $\phi(x, y, z)=\frac{q a b c}{4 \varepsilon_{0}} \int_{b}^{\infty} \int_{r(x, y, z ;),}^{\infty} \frac{f(s)}{\left(t+a^{2}\right)^{1 / 2}\left(t+b^{2}\right)^{1 / 2}\left(t+c^{2}\right)^{1 / 2}} d s d t$,

where the function $T(x, y, z ; t)$ is defined

(4) $T(x, y, z ; t) \equiv \frac{x^{2}}{t+a^{2}}+\frac{y^{2}}{t+b^{2}}+\frac{z^{2}}{t+c^{2}}$.

Using Eq. (3), the expressions for the field moments are computed to be

$$
\begin{aligned}
\left\langle x E_{x}\right\rangle & =\frac{\pi q a^{2} b^{2} c^{2} \Gamma(f)}{8 \varepsilon_{0}} a^{2} E_{C}^{I I}\left(c^{2}, b^{2}, a^{2}\right), & & \\
(5)\left\langle y E_{y}\right\rangle & =\frac{\pi q a^{2} b^{2} c^{2} \Gamma(f)}{8 \varepsilon_{0}} b^{2} E_{C}^{I I}\left(a^{2}, c^{2}, b^{2}\right), & & g(r) \equiv \int_{f}^{\infty} f(s) d s, \\
\left\langle z E_{z}\right\rangle & =\frac{\pi q a^{2} b^{2} c^{2} \Gamma(f)}{8 \varepsilon_{0}} c^{2} E_{C}^{I}\left(a^{2}, b^{2}, c^{2}\right), & & \Gamma(f) \equiv \int_{0}^{\infty} g^{2}\left(r^{2}\right) d r,
\end{aligned}
$$

where $\Gamma$ carries the distribution information (it is a function only of the profile $f$ ), and $E_{C} I l$ is the Carlson elliptic integral of the second kind [3].

Carlson's definitions for elliptic integrals are much more convenient to use in this situation. Moreover, they lend themselves nicely to numeric computation [4]. Definitions for these integrals are as follows: 


$$
\begin{aligned}
& E_{C}^{I}(x, y, z) \equiv \int_{0}^{\infty} \frac{d t}{(t+x)^{1 / 2}(t+y)^{1 / 2}(t+z)^{1 / 2}}, \\
& E_{C}^{I I}(x, y, z) \equiv \int_{0}^{\infty} \frac{d t}{(t+x)^{1 / 2}(t+y)^{1 / 2}(t+z)^{3 / 2}}, \\
& E_{C}^{I I}(x, y, z, p) \equiv \int_{0}^{\infty} \frac{d t}{(t+x)^{1 / 2}(t+y)^{1 / 2}(t+z)^{1 / 2}(t+p)} .
\end{aligned}
$$

Note that only $E_{C} I$ is symmetry in its arguments. It is possible to express all the conventional elliptic integrals $\left(E, F, K\right.$, etc.) in terms of $E_{C}{ }^{I}, E_{C}{ }^{I I}$ and $E_{C} I I I$.

As Sacherer discovered, the quantity $\Gamma(f)$ is almost constant. Therefore, we use its value for the uniform beam (which is $3 N^{2} / 10 \pi^{2} a^{2} b^{2} c^{2}$ ). Substituting this value, along with Eqs. (5), into equations (1) produces the complete set of equations

$$
\begin{array}{r}
X^{\prime \prime}+k_{x}^{2}(s) X-\frac{3 K}{8} X E_{C}^{I I}\left(Z^{2}, Y^{2}, X^{2}\right)-\frac{\varepsilon_{x}^{2}}{X^{3}}=0 \\
Y^{\prime \prime}+k_{y}^{2}(s) Y-\frac{3 K}{8} Y E_{C}^{I I}\left(X^{2}, Z^{2}, Y^{2}\right)-\frac{\varepsilon_{y}^{2}}{Y^{3}}=0 \\
Z^{\prime \prime}+k_{z}^{2}(s) Z-\gamma^{2} \frac{3 K}{8} Z E_{C}^{I I}\left(X^{2}, Y^{2}, Z^{2}\right)-\frac{\varepsilon_{z}^{2}}{Z^{3}}=0
\end{array}
$$

\subsection{Approximate Form of the Envelope Equation}

In the literature, the bunched beam envelope equations are usually expressed without explicit reference to elliptic integrals, typically by introduction of a "form factor" and/or approximations in lieu of the integrals. There, we see algebraic expressions instead of the special functions. One way to achieve this form is with the procedure given below.

Consider now the envelope equation for the $z$ plane. Writing out the elliptic integral explicitly in this case gives the following expression:

(8) $E_{C}^{I I}\left(X^{2}, Y^{2}, Z^{2}\right)=\frac{1}{(X Y)^{3 / 2}} \int_{0}^{\infty} \frac{d t}{(t+X / Y)^{1 / 2}(t+Y / X)^{1 / 2}\left(t+Z^{2} / X Y\right)^{3 / 2}}$,

where we have pulled a factor $X Y$ from each product in the denominator, then applied a change of variables. If we assume that, on average, the bunch $x$ envelope is approximately equal to the bunch $y$ envelope (i.e., the bunch is axis-symmetric on average) then $X / Y \approx Y / X \approx 1$. This approximation simplifies the space charge term to

(9) $\gamma^{2} \frac{3 K}{8} Z E_{C}^{I I}\left(X^{2}, Y^{2}, Z^{2}\right)=\gamma^{2} \frac{3 K}{8} \frac{1}{X Y} \xi\left(\frac{Z}{\sqrt{X Y}}\right)$,

where the function $\xi(\cdot)$ is typically called the form factor. Here it is given by

CKAllen_ICFA_Workshop.doc submitted to World Scientific 02/10/00: $6 / 20$ 
(10) $\xi(s) \equiv s \int_{0}^{\infty} \frac{d t}{(t+1)\left(t+s^{2}\right)^{3 / 2}}=\frac{2}{1-s^{2}} \begin{cases}1-\frac{s}{\sqrt{s^{2}-1}} \cosh ^{-1} s & \text { for } s^{2}>1 \\ 1-\frac{s}{\sqrt{1-s^{2}}} \cos ^{-1} s & \text { for } s^{2}<1\end{cases}$

A plot of this function is shown in Figure 2. From the figure we see that $\xi$ has a value of 2 at $s=0,2 / 3$ at $s=1$, then asymptotes toward zero as $s$ approaches infinity.

Substituting Eq. (9) into the envelope equations for the equivalent uniform ellipsoid, and using similar substitutions for the $x$ and $y$ planes, yields the approximate envelope equations. They appear as

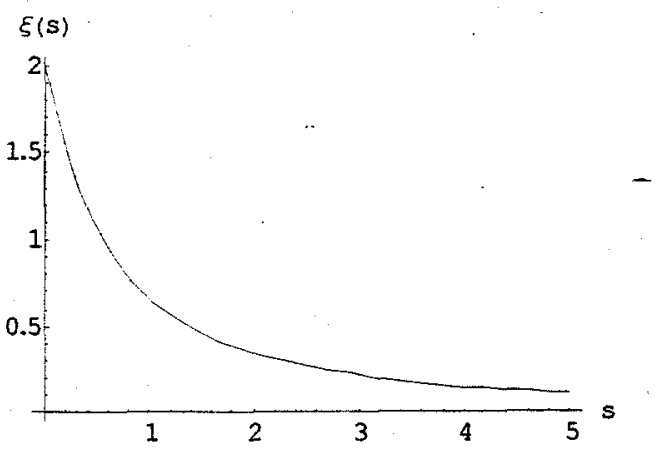

Figure 2: form function $\xi(s)$ follows:

$$
\begin{gathered}
X^{\prime \prime}+k_{x}^{2}(s) X-\frac{3 K}{8} \frac{\xi_{x}}{Y Z}-\frac{\varepsilon_{x}^{2}}{X^{3}}=0, \\
Y^{\prime \prime}+k_{y}^{2}(s) Y-\frac{3 K}{8} \frac{\xi_{y}}{X Z}-\frac{\varepsilon_{y}^{2}}{Y^{3}}=0 \\
Z^{\prime \prime}+k_{z}^{2}(s) Z-\gamma^{2} \frac{3 K}{8} \frac{\xi_{z}}{X Y}-\frac{\varepsilon_{z}^{2}}{Z^{3}}=0
\end{gathered}
$$

where the three form factors $\xi_{x}, \xi_{y}$, and $\xi_{z}$ are given by

$$
\xi_{x} \equiv \xi\left(\frac{X}{\sqrt{Y Z}}\right), \quad \xi_{y} \equiv \xi\left(\frac{Y}{\sqrt{X Z}}\right), \quad \xi_{z} \equiv \xi\left(\frac{Z}{\sqrt{X Y}}\right) .
$$

This is the approximation used by Bongardt $e t$ al [2] in their determination of bunch frequencies. We shall also use these equations for our analysis in the sequel.

\section{Bunch Oscillations}

Now that we have our modeling equations, we can compute the first-order oscillation frequencies and mode shapes for a bunched beam. Here we apply a perturbational approach to extract the lowest-order oscillation modes, assuming a given design. The first task is the construction of the perturbed equations.

CKAllen_ICFA_Workshop.doc submitted to World Scientific 02/10/00: $7 / 20$ 
The analysis of any system of ordinary differential equations can always be reduced to that of $n$ coupled, first-order equations [5]. This condition is achieved by transforming the system from the original dependent variables to state variables. However, because of the form of the envelope equations, the computational effort is reduced by expressing them in conservative form. They appear as

$$
\begin{array}{ll}
x^{\prime \prime}(s)=F[x(s), s] & x(0)=\alpha \\
x^{\prime}(0)=\beta
\end{array},
$$

where $x(s)$ and $F(x, s)$ are vector quantities defined by

$$
x(s) \equiv\left(\begin{array}{c}
X(s) \\
Y(s) \\
Z(s)
\end{array}\right), F(x, s) \equiv\left(\begin{array}{c}
-k_{x}^{2}(s) X+\frac{3 K}{8} \frac{\xi_{x}(s)}{Y Z}+\frac{\varepsilon_{x}^{2}}{X^{3}} \\
-k_{y}^{2}(s) Y+\frac{3 K}{8} \frac{\xi_{y}(s)}{X Z}+\frac{\varepsilon_{y}^{2}}{Y^{3}} \\
-k_{z}^{2}(s) Z+\gamma^{2} \frac{3 K}{8} \frac{\xi_{z}(s)}{X Y}+\frac{\varepsilon_{z}^{2}}{Z^{3}}
\end{array}\right) .
$$

The vectors $\alpha$ and $\beta$ represent the initial values. The vector $x(s)$ is our state variable and the function $F(x, s)$ is known as the characteristic function.

\subsection{Perturbation Analysis}

In the theory of perturbations, one initially has a known solution to Eqs. (13), one that is presumably easy to find. Then Eq. (13) is expanded in a perturbation parameter about the known solution. The perturbation parameter may or may not have physical interpretation, it functions primarily as a bookkeeper to identify the subsystems of various orders [8]. Typically, the subsystems are solvable by standard analytic techniques, however, they become increasingly more complicated as the order increases.

Assume that our system depends continuously on $\varepsilon$, the perturbation parameter. This implies that the characteristic function has the form $F=F(x, s ; \varepsilon)$. Consequently, the state variable also depends continuously on $\varepsilon$ (i.e., $x=x(s ; \varepsilon)$ ). When $\varepsilon=0$ we choose $F(x, s ; \varepsilon)$ to select the smooth beam approximation to the envelope equations. Then as $\varepsilon$ approaches unity the system approaches the true dynamic case.

One way to achieve this condition, is to expand the focusing functions in $F$ as

$$
k_{i}^{2}(s)=k_{i, 0}^{2}+\varepsilon k_{i, 1}^{2}(s)+\varepsilon^{2} k_{i, 2}^{2}(s)+\varepsilon^{3} k_{i, 3}^{2}(s)+\ldots \quad \text { for } i \in\{x, y, z\},
$$

where the $k_{0, i}$ are the constants representing the average focusing of the smooth beam approximation. Thus, at $\varepsilon=0$, we have the smooth beam approximation. The remaining terms in the expansion are all functions of $s$ and account for the variation

CKAllen_ICFA_Workshop.doc submitted to World Scientific 02/10/00: $8 / 20$ 
in focusing along the design trajectory. Likewise, the solution $x(s ; \varepsilon)$ can now be expanded in the series

$$
x(s ; \varepsilon)=x_{0}(s)+x_{1}(s)+\varepsilon^{2} x_{2}(s)+\varepsilon^{3} x_{3}(s)+\ldots,
$$

so that $x_{n}(s)=n ! \partial^{n} x(s ; \varepsilon) / \partial \varepsilon^{n}$. At $\varepsilon=0$, we have $x(s ; \varepsilon)=x_{0}(s)$ which, again, shall represent the smooth beam solution to the bunched beam envelope equations.

To form the differential systems for the perturbed solutions, we assemble the original state equations then start taking derivatives of everything in sight with respect to $\varepsilon$. Collecting the terms involving the various powers of $\varepsilon$ yields differential systems for the various solution orders. The first three systems are

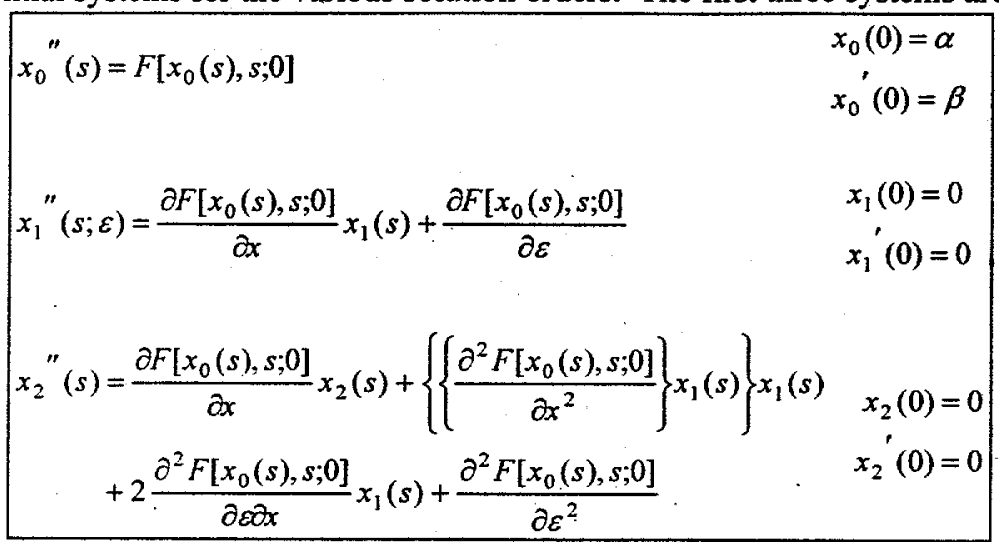

Note that $\partial F / \partial x$ is a square matrix and $\partial^{2} F / \partial x^{2}$ is a rank-three tensor. Also notice that these systems are all linear except for the first one (the zero-order system).

\subsection{Zero-Order (Smooth Beam) Solution}

To find $x_{0}$ we need to solve the zero-order system in Eqs. (17). Since $F(x, s ; \varepsilon)$ is independent of $s$ at $\varepsilon=0, x_{0}$ must also be independent of $s$ at $\varepsilon=0$ (i.e., $x_{0}$ is a constant). Consequently, $x_{0}{ }^{\prime}=x_{0}{ }^{\prime \prime}=0$ and $x_{0}$ solves the system

(18) $F\left[x_{0}, s ; 0\right]=0$.

This vector equation contains a set of three independent, nonlinear, algebraic equations involving the matched beam envelopes, which we denote $X_{0}, Y_{0}$ and $Z_{0}$. We shall assume, however, that the $x$ and $y$ envelopes are designed to have an equal average value $R_{0}$, that is,

(19) $X_{0}=Y_{0}=R_{0}$.

With the assumption it is possible to solve for the zero-order focusing constants $k_{0, r}$ and $k_{0, z}$ in terms of the envelopes $R_{0}$ and $Z_{0}$. The results are

CKAllen_ICFA_Workshop.doc submitted to World Scientific 02/10/00: 9/20 


$$
k_{r, 0}{ }^{2}=\frac{3 K \xi_{r}}{8 Z_{0} R_{0}^{2}}+\frac{\varepsilon_{r}^{2}}{R_{0}^{4}}, \quad \text { and } \quad k_{z, 0}^{2}=\gamma^{2} \frac{3 K \xi_{z}}{8 Z_{0} R_{0}^{2}}+\frac{\varepsilon_{z}^{2}}{Z_{0}^{4}} .
$$

Thus, if we have a design where the average bunch radius is $R_{0}$ and average bunch length is $Z_{0}$ then the above values represent the average focusing necessary to maintain that beam size.

These results may also be expressed in terms of the single-particle phase advances per unit length. Recall that $k_{r, 0}$ and $k_{z, 0}$ denote the radial and longitudinal phase advances without space charge. Now introducing $k_{r}$ and $k_{z}$ as the radial and longitudinal phase advances (per unit length) with space charge, we have

$$
\begin{array}{ll}
k_{r}^{2}=\frac{\varepsilon_{r}^{2}}{R_{0}^{4}}, & k_{z}^{2}=\frac{\varepsilon_{z}^{2}}{Z_{0}^{4}}, \\
k_{r, 0}^{2}=k_{r}^{2}+\frac{3 K \xi_{r}}{8 Z_{0} R_{0}^{2}}, & k_{z, 0}^{2}=k_{z}^{2}+\frac{3 K \gamma^{2} \xi_{z}}{8 Z_{0} R_{0}^{2}} .
\end{array}
$$

\subsection{First-Order System}

Consider now the second differential system in Eqs. (17). It describes the firstorder variation of the smooth beam approximation. Note here that the perturbed focusing strengths $k_{r, 1}$ and $k_{z, 1}$ are functions of the path-length parameter $s$. The first-order system in (17) can be written more compactly as

$$
\begin{array}{rr}
x_{1}{ }^{\prime}(s)+A(s) x_{1}(s)=u(s), & x_{1}(0)=\xi, \\
& x_{1}^{\prime}(0)=\xi^{\prime},
\end{array}
$$

where $\xi$ and $\xi$ account for any initial perturbations at $s=0$. The function $u(s)$ is the "control" of the system. Assuming quadrupole-focusing symmetry, it is defined by

$$
u(s) \equiv \frac{\partial F\left[x_{0}, s ; 0\right]}{\partial \varepsilon}=\left(-k_{r, 1}^{2}(s) R_{0}+k_{r, 1}^{2}(s) R_{0}-k_{z, 1}^{2}(s) Z_{0}\right)^{T} .
$$

The matrix $A(s)$ is defined

$$
{ }^{(24)} A(s)=\frac{\partial F\left[x_{0}, s ; 0\right]}{\partial x}=\left(\begin{array}{ccc}
\left(k_{r, 0}^{2}+3 k_{r}^{2}+2 \delta_{r}^{2}\right) & \left(k_{r, 0}^{2}-k_{r}^{2}-\delta_{r}^{2}\right) & \frac{R_{0}}{Z_{0}}\left(k_{r, 0}^{2}-k_{r}^{2}-\delta_{r}^{2}\right) \\
\left(k_{r, 0}^{2}-k_{r}^{2}-\delta_{r}^{2}\right) & \left(k_{r, 0}^{2}+3 k_{r}^{2}+2 \delta_{r}^{2}\right) & \frac{R_{0}}{Z_{0}}\left(k_{r, 0}^{2}-k_{r}^{2}-\delta_{r}^{2}\right) \\
\frac{Z_{0}}{R_{0}}\left(k_{z, 0}^{2}-k_{z}^{2}-\delta_{z}^{2}\right) & \frac{Z_{0}}{R_{0}}\left(k_{z, 0}^{2}-k_{z}^{2}-\delta_{z}^{2}\right) & \left(k_{z, 0}^{2}+3 k_{z}^{2}+2 \delta_{z}^{2}\right)
\end{array}\right) \text {. }
$$

where we have introduced the phase advance perturbation parameters

CKAllen_ICFA_Workshop.doc submitted to World Scientific 02/10/00: 


$$
\begin{array}{lll}
\delta_{r}^{2} \equiv-\frac{3 K \xi_{r}^{\prime}}{16 R_{0}^{3 / 2} Z_{0}^{3 / 2}}, & \text { where } & \left.\xi_{r}^{\prime} \equiv \frac{d \xi(\sigma)}{d \sigma}\right|_{\sigma=\sqrt{\frac{R_{0}}{z_{0}}}} \\
\delta_{z}^{2} \equiv-\frac{3 K \gamma^{2} \xi_{z}^{\prime}}{16 R_{0}^{3}}, & \left.\xi_{z}^{\prime} \equiv \frac{d \xi(\sigma)}{d \sigma}\right|_{\sigma=\frac{z_{0}}{R_{0}}} .
\end{array}
$$

The $s$ dependence of the derivative matrix $A$ arises implicitly from the beam parameters $K, \varepsilon_{r}, \varepsilon_{z}, R_{0}, Z_{0}$ and $\gamma$. Typically this variation is quite slow compared to the length of a focusing/accelerating period $S$. Consequently, considering $A$ constant over a period length is a reasonable approximation.

We further simplify $A$ by defining the modified phase advances

$$
\begin{aligned}
& K_{r}^{2} \equiv k_{r, 0}^{2}-k_{r}^{2}-\delta_{r}^{2}, \\
& K_{z}^{2} \equiv k_{z, 0}^{2}-k_{z_{i}}^{2}-\delta_{z}^{2},
\end{aligned}
$$

and

$$
\begin{aligned}
& \theta_{r}^{2} \equiv k_{r, 0}^{2}+k_{r}^{2}+\frac{1}{2} \delta_{r}^{2}, \\
& \theta_{z}^{2} \equiv \frac{1}{2} k_{z, 0}^{2}+\frac{3}{2} k_{z}^{2}+\delta_{z}^{2} .
\end{aligned}
$$

With these definitions $A$ appears more compactly as

$$
A=\left(\begin{array}{ccc}
2 \theta_{r}^{2}-K_{r}^{2} & K_{r}^{2} & \frac{R_{0}}{Z_{0}} K_{r}^{2} \\
K_{r}^{2} & 2 \theta_{r}^{2}-K_{r}^{2} & \frac{R_{0}}{Z_{0}} K_{r}^{2} \\
\frac{Z_{0}}{R_{0}} K_{z}^{2} & \frac{Z_{0}}{R_{0}} K_{z}^{2} & 2 \theta_{z}^{2}
\end{array}\right) .
$$

Letting $A(s)=A$, a constant, the solution to (22) is the convolution equation

$$
x_{1}(s)=\cos (\sqrt{A} s) \xi+[\sqrt{A}]^{-1} \sin (\sqrt{A} s) \xi^{\prime}+\int_{0}^{\infty}[\sqrt{A}]^{-1}[\sin (\sqrt{A}(s-\sigma)] u(\sigma) d \sigma,
$$

where the matrices $\sin (\sqrt{ } A s)$ and $\cos (\sqrt{ } A s)$ are typically defined in terms of the cosine and sine Taylor series, respectively (likewise for the square root function). Most of the first-order bunch characteristics can be inferred from matrix $\sin \left(\vee_{A s}\right)$.

\subsubsection{Bunch Oscillation Frequencies}

The matrix $\sin \left(\vee_{A s}\right)$ represents the first-order impulse response of the bunch and contains all its natural oscillation modes. Specifically, the eigenvalues of $\sin \left(\vee_{A s}\right)$ are the natural oscillation frequencies of the bunch while the eigenvectors are the

CKAllen_ICFA_Workshop.doc submitted to World Scientific 02/10/00: 
oscillation mode shapes. Consequently, $A$, in turn, contains the same information. To see this, assume all eigenvalues are distinct and thus $A$ can be diagaonalized as

$$
A=P \Lambda P^{-1}=P\left(\begin{array}{ccc}
\lambda_{1} & 0 & 0 \\
0 & \lambda_{2} & 0 \\
0 & 0 & \lambda_{3}
\end{array}\right) P^{-1}
$$

where the $\lambda_{i}$ are eigenvalues of $A$ and $P$ is the augmented matrix of eigenvectors (i.e., the diagonalization matrix). Then the trigonometric matrix functions are given according to the prescription

$$
\sin (\sqrt{A} s)=P \sin (\sqrt{\Lambda} s) P^{-1}=P\left(\begin{array}{ccc}
\sin \sqrt{\lambda_{1}} s & 0 & 0 \\
0 & \sin \sqrt{\lambda_{2}} s & 0 \\
0 & 0 & \sin \sqrt{\lambda_{3}} s
\end{array}\right) P^{-1},
$$

Thus, we see that the natural oscillation phase advances of the bunch are simply the roots of the eigenvalues. We find that, in general, they are all distinct and, thus, there are three separate modal frequencies for bunch oscillations. The eigenvalues, and consequent phase advances, are

$$
\begin{aligned}
& k_{Q} \equiv \sqrt{\lambda_{1}}=\left[2 \theta_{r}^{2}-2 K_{r}^{2}\right]^{1 / 2}=2\left[k_{r}^{2}+\frac{3}{4} \delta_{r}^{2}\right]^{1 / 2}, \\
& k_{H} \equiv \sqrt{\lambda_{2}}=\left[\left(\theta_{r}^{2}+\theta_{z}^{2}\right)+\sqrt{\left(\theta_{r}^{2}-\theta_{z}^{2}\right)^{2}+2 K_{r}^{2} K_{z}^{2}}\right]^{1 / 2}, \\
& k_{L} \equiv \sqrt{\lambda_{3}}=\left[\left(\theta_{r}^{2}+\theta_{z}^{2}\right)-\sqrt{\left(\theta_{r}^{2}-\theta_{z}^{2}\right)^{2}+2 K_{r}^{2} K_{z}^{2}}\right]^{1 / 2} .
\end{aligned}
$$

In the above we have explicitly defined the three natural phase advances of the bunch, $k_{Q}, k_{H}$, and $k_{L}$ in terms of the eigenvalues $\lambda_{1}, \lambda_{2}$, and $\lambda_{3}$.

We see that there is one pure quadrupole mode and two fully coupled modes. In Bongardt et al's treatment, they did not include the $\delta_{r}$ and $\delta_{z}$ terms in their expansions. These terms contribute significantly to the values of $k_{Q}, k_{H}$, and $k_{L}$, usually about $10 \%$. If we neglect these terms, we obtain results that are similar to their findings, however our values still differ in the factor 2 seen under the radical in eigenfrequencies $k_{Q}, k_{H}$, and $k_{L}$ [2].

\subsubsection{Bunch Oscillation Modes}

The mode shapes are determined by inspecting the eigenvectors of $A$, since $\sin (\sqrt{A} s)$ has the same eigenvectors as $A$. The eigenvector corresponding to $k_{Q}$ is

$$
e_{Q}=\left(\begin{array}{lll}
1 & -1 & 0
\end{array}\right)^{T} \text {. }
$$

CKAllen_ICFA_Workshop.doc submitted to World Scientific 02/10/00: 
As expected, this vector forms the pure quadrupole mode and is completely decoupled from the longitudinal behavior. The next eigenvector has the form

$$
e_{H}=\left(\begin{array}{lll}
1 & 1 & r_{H}
\end{array}\right)^{T}
$$

where $r_{H}$ is a positive constant (see below). This eigenvector forms the "breathing mode" for the bunch where all planes oscillate in phase. This mode is also called the "high mode". Finally, the last eigenvector $e Q$ appears as

$$
e_{Q}=\left(\begin{array}{lll}
1 & 1 & -r_{L}
\end{array}\right)^{T}
$$

where $r_{L}$ is another positive constant. This eigenvector forms the axisymmetric mode known as the "low mode". Here the radial and longitudinal components oscillate 180 degrees out of phase. The values of coupling constants $r_{H}$ and $r_{L}$ are

$$
\begin{aligned}
& r_{H} \equiv \frac{Z_{0}}{R_{0}} \frac{-\left(\theta_{r}^{2}-\theta_{z}^{2}\right)+\sqrt{\left(\theta_{r}^{2}-\theta_{z}^{2}\right)^{2}+2 K_{r}^{2} K_{z}^{2}}}{K_{r}^{2}} \\
& r_{L} \equiv \frac{Z_{0}}{R_{0}} \frac{\left(\theta_{r}^{2}-\theta_{z}^{2}\right)+\sqrt{\left(\theta_{r}^{2}-\theta_{z}^{2}\right)^{2}+2 K_{r}^{2} K_{z}^{2}}}{K_{r}^{2}} .
\end{aligned}
$$

Note that $r_{H}<r_{L}$.

Now we reconsider the convolution equation (29). First, it is convenient to transform the system into the eigencoordinates of $A$. Let the new state variables be

$$
\begin{aligned}
& y(s) \equiv P^{-1} x_{1}(s), \\
& \eta \equiv P^{-1} \xi, \\
& \eta^{\prime} \equiv P^{-1} \xi^{\prime},
\end{aligned}
$$

From the expressions for the eigenvectors of $A$ we know that the diagonalization matrix $P$ has the form

$$
P=\left(\begin{array}{ccc}
1 & 1 & 1 \\
-1 & 1 & 1 \\
0 & r_{H} & -r_{L}
\end{array}\right)
$$

and, therefore,

$$
P^{-1}=\frac{1}{2\left(r_{L}+r_{H}\right)}\left(\begin{array}{ccc}
\left(r_{L}+r_{H}\right) & -\left(r_{L}+r_{H}\right) & 0 \\
r_{L} & r_{L} & 2 \\
r_{H} & r_{H} & -2
\end{array}\right) .
$$

Using $P^{-1}$, we find that the transformed control $v(s)$ has the form

CKAllen_ICFA_Workshop.doc submitted to World Scientific 02/10/00: 


$$
v(s)=\left(\begin{array}{c}
-k_{r, 1}^{2}(s) R_{0} \\
-k_{z, 1}^{2}(s) \frac{Z_{0}}{r_{H}+r_{L}} \\
k_{z, 1}^{2}(s) \frac{Z_{0}}{r_{H}+r_{L}}
\end{array}\right) .
$$

In the eigencoordinates, first position corresponds to the quadrupole mode, second to the high mode, and the last corresponds to the low mode. Thus, to first order, we are unable to excite anything but the quadrupole mode with transverse focusing.

Using the eigencoordinate transform, the convolution equation decouples into

$$
\begin{aligned}
& Q(s)=\cos \left(k_{Q} s\right) Q_{0}+\frac{1}{k_{Q}} \sin \left(k_{Q} s\right) Q_{0}^{\prime}-\frac{R_{0}}{k_{Q}} \int \sin k_{Q}(s-\sigma) k_{r, 1}^{2}(\sigma) d \sigma, \\
& H(s)=\cos \left(k_{H} s\right) H_{0}+\frac{1}{k_{H}} \sin \left(k_{H} s\right) H_{0}^{\prime}-\frac{Z_{0}}{k_{H}\left(r_{H}+r_{L}\right)} \int_{0} \sin k_{H}(s-\sigma) k_{z, 1}^{2}(\sigma) d \sigma, \\
& L(s)=\cos \left(k_{L} s\right) L_{0}+\frac{1}{k_{L}} \sin \left(k_{L} s\right) L_{0}^{\prime}+\frac{Z_{0}}{k_{L}\left(r_{H}+r_{L}\right)} \int \sin k_{L}(s-\sigma) k_{z, 1}^{2}(\sigma) d \sigma,
\end{aligned}
$$

where $Q(s), H(s)$, and $L(s)$ are the first-order quadrupole, high, and low mode amplitudes, respectively. The constants $Q_{0}, Q_{0}{ }^{\prime}, H_{0}, H_{0}{ }^{\prime}, L_{0}$, and $L_{0}$ ' represent any initial excitations of the bunch modes at point $s=0$.

\subsubsection{Mode Excitations for AG Focusing}

Here we investigate the first-order response of the beam bunch to the focusing structures in an accelerator/transport system. That is we wish to determine the firstorder perturbations $x_{1}=\left(X_{1}, Y_{1}, Z_{1}\right)$ of the bunch envelopes propagating through the accelerator and/or transport system. Assuming the machine is periodic in $s$ with period $S$, the focusing functions $k_{i, 1}{ }^{2}(s)$ can be expanded into a Fourier series. Moreover, we assume, as before, that the focusing is AG in nature. If so, we can pick a coordinate origin in $s$ so that $k_{r, 1}{ }^{2}(s)$ is an odd function and $k_{z, 1}{ }^{2}(s)$ is an even function. This condition yields the following expansions

$$
\begin{aligned}
& k_{r, 1}^{2}(s)=\sum_{n=1}^{\infty} b_{n} \sin \left(n k_{m} s\right), \\
& k_{z, 1}^{2}(s)=\sum_{n=0}^{\infty} a_{n} \cos \left(n k_{m} s\right),
\end{aligned}
$$

and

CKAllen_ICFA_Workshop.doc submitted to World Scientific 02/10/00: 


$$
\begin{array}{lll}
a_{0}=\frac{1}{S} \int_{0}^{S} k_{z, 1}^{2}(s) d s, & a_{n}=\frac{2}{S} \int_{0}^{S} k_{z, 1}^{2}(s) \cos \left(n k_{m} s\right) d s & \text { for } n=1,2,3, \ldots \\
b_{0}=0, & b_{n}=\frac{2}{S} \int_{b}^{S} k_{r, 1}^{2}(s) \sin \left(n k_{m} s\right) d s & \text { for } n=1,2,3, \ldots
\end{array}
$$

Inserting the expanded expressions for the focusing into the equations for mode amplitudes yields

$$
\begin{aligned}
& Q(s)=R_{0} \sum_{n=1}^{\infty} b_{n} \frac{k_{Q} \sin \left(n k_{m} s\right)-n k_{m} \sin \left(k_{Q} s\right)}{k_{Q}\left(n^{2} k_{m}^{2}-k_{Q}^{2}\right)}, \\
& H(s)=R_{0} \frac{K_{r}^{2}}{2 \sqrt{\left(\theta_{r}^{2}-\theta_{z}^{2}\right)^{2}+2 K_{r}^{2} K_{z}^{2}}} \sum_{n=0}^{\infty} a_{n} \frac{\cos \left(n k_{m} s\right)-\cos \left(k_{H} s\right)}{n^{2} k_{m}^{2}-k_{H}^{2}}, \\
& L(s)=-R_{0} \frac{K_{r}^{2}}{2 \sqrt{\left(\theta_{r}^{2}-\theta_{z}^{2}\right)^{2}+2 K_{r}^{2} K_{z}^{2}}} \sum_{n=0}^{\infty} a_{n} \frac{\cos \left(n k_{m} s\right)-\cos \left(k_{L} s\right)}{n^{2} k_{m}^{2}-k_{L}^{2}},
\end{aligned}
$$

where we have expanded the mode ratios $r_{H}$ and $r_{L}$ and we have assumed that the initial mode amplitudes are zero (i.e., $Q_{0}=0, H_{0}=0$, etc.) The response has frequency components of the machine along with the natural modes of the bunch. So long as the natural frequencies of the bunch are sufficiently separated from the machine frequencies, the bunch is stable. That is, we require that

$$
\frac{1}{n^{2} k_{m}^{2}-k_{\alpha}^{2}}<1
$$

where $\alpha$ is either $Q, H$, or $L$. Note that the quadrupole mode is more sensitive to the machine harmonics. The quadrupole amplitude falls off as $O(1 / n)$ with increasing $n$ harmonics, whereas the high and low modes fall off as $O\left(1 / n^{2}\right)$.

To convert back to the standard state variable components $X_{1}, Y_{1}, Z_{1}$ we simply premultiply the above results with the diagonalization matrix $P$. The result is

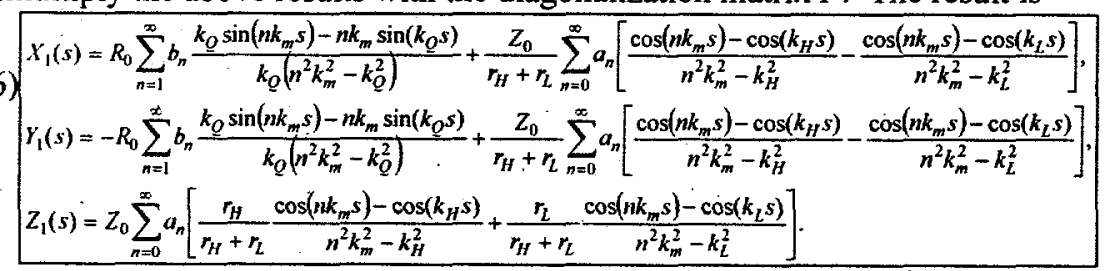

\section{Halo Formation}

Now we begin to briefly outline our current investigations into halo formation using the perturbation analysis. As mentioned in the introduction, we have employed two approaches in this investigation. The two approaches differ in the manner in which they treat the space charge coupling between core and particle. In one, we directly

CKAllen_ICFA_Workshop.doc submitted to World Scientific 02/10/00: 
expand the expression for the potential of an ellipsoidally symmetric distribution. In the other approach, we first expand the self fields as a polynomial in the coordinates $(x, y, z)$. The coefficients of the polynomial are functions of the bunch envelopes $X, Y$, and $Z$.

First, we cover the techniques that are common to both approaches. Specifically, the equations of motion are introduced, along with the appropriate expansions for the particle trajectories. We also discuss the multiple-scale expansion in terms of the independent variable $s$. Last, the equations of motion are rewritten in the partially expanded form so to be accessible to either technique.

\subsection{The Common Expansions}

The equations of motion for the $x, y, z$ coordinates of a particle may be written

$$
\begin{aligned}
& x^{\prime \prime}+k_{x}^{2}(s) x=q E_{x}(x, y, z, X, Y, Z ; s), \\
& y^{\prime \prime}+k_{y}^{2}(s) y=q E_{y}(x, y, z, X, Y, Z ; s), \\
& z^{\prime \prime}+k_{z}^{2}(s) z=q E_{z}(x, y, z, X, Y, Z ; s),
\end{aligned}
$$

where the $E_{\alpha}$ are the self electric-field components in the respective coordinate directions. As indicated, the electric fields provided the coupling between the particle motion and the core envelope oscillations. Success of the analysis depends greatly on the method and accuracy achieved when expanding these fields.

Oscillations of the beam envelope are the primary mechanism behind halo formation. Consequently, they constitute the known input to the system. The bunch envelopes $X(s), Y(s)$, and $Z(s)$.make small oscillations about their nominal values of $R_{0}$ and $Z_{0}$. Thus, the envelopes have the form

$$
\begin{aligned}
& X(s)=R_{0}+\varepsilon X_{1}(s), \\
& Y(s)=R_{0}+\varepsilon Y_{1}(s), \\
& Z(s)=Z_{0}+\varepsilon Z_{1}(s) .
\end{aligned}
$$

where the perturbation parameter $\varepsilon$ is now recognized as the envelope oscillation amplitude. From the previous analysis we know that the first order envelope variations $X_{1}, Y_{1}, Z_{1}$ have the form in Eqs. (46).

Now we begin expanding trajectories in the amplitude factor $\varepsilon$. Assume that the solutions to the equations of motion can be expanded as

$$
\begin{aligned}
& x(s)=x_{0}(s)+\varepsilon x_{1}(s)+\varepsilon^{2} x_{2}(s)+O\left(\varepsilon^{3}\right), \\
& y(s)=y_{0}(s)+\varepsilon y_{1}(s)+\varepsilon^{2} y_{2}(s)+O\left(\varepsilon^{3}\right), \\
& z(s)=z_{0}(s)+\varepsilon z_{1}(s)+\varepsilon^{2} z_{2}(s)+O\left(\varepsilon^{3}\right),
\end{aligned}
$$

where the $x_{n}, y_{n}$, and $z_{n}$ are the solution components of order $n$. Thus, $x_{0}, y_{0}, z_{0}$ are the zero-order solutions which the higher ordered solutions $(n=1,2,3, \ldots)$ orbit. If

CKAllen_ICFA_Workshop.doc submitted to World Scientific 02/10/00: 
we were to simply substitute these expansions back into the equations of motion, we would obtain solutions containing so-called secular terms. Such terms cause the expanded solution to diverge from the true solution as $s$ increases. This situation arises whenever the form of the expansion is insufficient to accurately represent the true solutions over an extended interval of $s$.

\subsection{Multiple Time Scales}

The appearance of secular terms can be circumvented by introducing additional degrees of freedoms. Specifically, consider multiple time scales of the form

(50) $s_{i} \equiv \varepsilon^{i} s$.

To carry out the expansion to second order in $\varepsilon$, we need to retain $s_{0}, s_{1}$ and $s_{2}$. Thus, in the equations of motion differentiation with respect to the path length $s$ now has the form

$$
\frac{d}{d s}=\frac{\partial}{\partial s_{0}}+\varepsilon \frac{\partial}{\partial s_{1}}+\varepsilon^{2} \frac{\partial}{\partial s_{2}},
$$

$$
\frac{d^{2}}{d s^{2}}=\frac{\partial^{2}}{\partial s_{0}^{2}}+2 \varepsilon \frac{\partial^{2}}{\partial s_{0} \partial s_{1}}+\varepsilon^{2}\left(2 \frac{\partial^{2}}{\partial s_{0} \partial s_{2}}+\frac{\partial^{2}}{\partial s_{1}^{2}}\right) \text {. }
$$

The variables $s_{0}, s_{1}$ and $s_{2}$ now represent increasingly slower time scales.

We now collect the results of this section to identify the expanded trajectory equations. For the $x$ plane, the first three perturbation equations are given as

$$
\begin{aligned}
& \partial_{0}^{2} x_{0}+k_{x, 0}^{2} x_{0}=O_{0}\left(q E_{x}\right), \\
& \partial_{0}^{2} x_{1}+k_{x, 0}^{2} x_{1}=-2 \partial_{1} \partial_{0} x_{0}-k_{x, 1}^{2}(s) x_{0}+O_{1}\left(q E_{x}\right) \\
& \partial_{0}^{2} x_{2}+k_{x, 0}^{2} x_{2}=-\partial_{1}^{2} x_{0}-2 \partial_{0} \partial_{2} x_{0}-k_{x, 2}^{2}(s) x_{0}-2 \partial_{1} \partial_{0} x_{1}-k_{x, 1}^{2}(s) x_{1}+O_{2}\left(q E_{x}\right),
\end{aligned}
$$

where the shorthand $\partial_{0}, \partial_{1}$ and $\partial_{2}$ denotes the partials $\partial / \partial s_{0}, \partial / \partial s_{1}$ and $\partial / \partial s_{2}$, respectively. The operator $O_{n}\left(E_{x}\right)$ selects the term in the expansion of $E_{x}$ containing the coefficient $z^{n}$. The expanded equations of motion for the $y$ and $z$ planes are analogous to the above set. Note that the left-hand side of each equation constitutes a linear differential operator. The coupling between solution orders occurs on the right-hand side, which contains terms involving all the lower ordered solutions. Thus, the equations for the higher-order components of the trajectories become increasingly more complicated.

\subsection{Approach One: Direct Expansion of the Self-Fields}

Here, the self-fields depend upon the specific form of the distribution, represented by the function $f$. Therefore, this function must also expanded in the perturbation parameter $\varepsilon$ according to

CKAllen_ICFA_Workshop.doc submitted to World Scientific 02/10/00: 


$$
f(t)=f_{0}(t)+\varepsilon f_{1}(t)+\varepsilon^{2} f_{2}(t)+O\left(\varepsilon^{3}\right) .
$$

To make calculations possible we assume that $f_{0}$ is the uniform distribution. Consequently, particle behavior in response to nonuniformities will be seen in the first-order solutions $x_{1}, y_{1}, z_{1}$, and higher. This situation is consistent with a space charge dominated beam [10].

Note that after substituting Eqs. (48), Eqs. (49), and Eq. (53) into Eqs. (3), it is possible to consider the field components as functions of $\varepsilon$. By Taylor expanding in $\varepsilon$ about $\varepsilon=0$, the fields in each plane $\alpha \in\{x, y, z\}$ are expanded in the form

$$
E_{\alpha}(\varepsilon)=E_{\alpha, 0}(0)+\varepsilon E_{\alpha, 1}^{\prime}(0)+\varepsilon^{2} \frac{1}{2} E_{\alpha, 2}{ }^{\prime \prime}(0)+O\left(\varepsilon^{3}\right),
$$

where the prime indicates differentiation in $\varepsilon$. For the $x$ plane this procedure yields

$$
\begin{aligned}
E_{x} & =I_{2,1 / 2}\left(f_{0}\right) x_{0} \\
& +\varepsilon I_{2,1 / 2}\left(f_{1}\right) x_{0}+\varepsilon I_{2,1 / 2}\left(f_{0}\right) x_{1} \\
& -\varepsilon\left[I_{3,1 / 2}\left(f_{0}\right) R_{0}\left(3 X_{1}+Y_{1}\right)+I_{2,3 / 2}\left(f_{0}\right) Z_{0} Z_{1}\right] x_{0} \\
& +2 \varepsilon I_{3,1 / 2}\left(f_{0}^{\prime}\right)\left(x_{0}^{2} x_{1}+x_{0} y_{0} y_{1}\right)+2 \varepsilon I_{2,3 / 2}\left(f_{0}^{\prime}\right) x_{0} z_{0} z_{1} \\
& -2 \varepsilon I_{4,1 / 2}\left(f_{0}^{\prime}\right) R_{0}\left(X_{1} x_{0}^{3}+Y_{1} x_{0} y_{0}^{2}\right)-2 \varepsilon I_{2,5 / 2}\left(f_{0}^{\prime}\right) Z_{0} Z_{1} x_{0} z_{0}^{2} \\
& +O\left(\varepsilon^{2}\right)
\end{aligned}
$$

where $l_{p, q}(f)$ denotes the integral

$$
I_{i, j}(f) \equiv \frac{Q}{4 \pi \varepsilon_{0} F_{1 / 2}} \int_{0}^{\infty}\left[f\left(\frac{x_{0}^{2}+y_{0}^{2}}{t+R_{0}^{2}}+\frac{z_{0}^{2}}{t+Z_{0}^{2}}\right)\left(t+R_{0}^{2}\right)^{p}\left(t+Z_{0}^{2}\right)^{q}\right] d t .
$$

Obviously this approach is simple but leaves us with complex expression to deal with. Employing the Fredholm alternative theorem here can be complicated.

\subsection{Approach Two: A Weighted Expansion of Self-Fields}

Here we assume that the self-fields may be accurately represented with a polynomial expansion in the Cartesian coordinate variables $x, y, z$. Considering only the $x$ axis dynamics we have for the $x$ component of the electric field

$$
E_{x}(x) \approx a_{0}+a_{1} x+a_{2} x^{2}+a_{3} x^{3}+a_{4} x^{4}+O\left(x^{5}\right),
$$

where the $a_{n}$ are typically functions of the bunch envelopes. In general, $E_{x}$ may also be a function of $y$ and $z$, however, the assumption of ellipsoidal symmetry eliminates this possibility. Now we are relegated to the determination of the proper $a_{n}$ 's. One simple method is to Taylor expand the electric field component $E_{x}$. With this choice for the $a_{n}$, the expansion is only accurate near the coordinate origin. Since the dynamics leading to halo creation occur throughout the distribution, this

CKAllen_ICFA_Workshop.doc submitted to World Scientific 02/10/00: 
expansion is completely inadequate. We require an expansion that is meaningful in the entire region of the beam including the halo region.

We are unlikely to find a polynomial expansion that closely approximates the true fields over the entire bunch region. However, it is possible to find one that represents the bunch fields in an averaged sense (i.e., in a Lebesgue space) over the beam. The idea is to take a weighted projection of the true fields onto the function space spanned by basis $\left\{1, x, x^{2}, x^{3}, x^{4}\right\}$. Letting $\langle\cdot, \cdot\rangle$ be the weighted inner product, this criterion translates into the following equation for coefficients $a_{n}$ :

$$
\left(\begin{array}{cccc}
\langle 1,1\rangle & \langle 1, x\rangle & \left\langle 1, x^{2}\right\rangle & \left\langle 1, x^{3}\right\rangle \\
\langle x, 1\rangle & \langle x, x\rangle & \left\langle x, x^{2}\right\rangle & \left\langle x, x^{3}\right\rangle \\
\left\langle x^{2}, 1\right\rangle & \left\langle x^{2}, x\right\rangle & \left\langle x^{2}, x^{2}\right\rangle & \left\langle x^{2}, x^{3}\right\rangle \\
\left\langle x^{3}, 1\right\rangle & \left\langle x^{3}, x\right\rangle & \left\langle x^{3}, x^{2}\right\rangle & \left\langle x^{3}, x^{3}\right\rangle
\end{array}\right)\left(\begin{array}{l}
a_{0} \\
a_{1} \\
a_{2} \\
a_{3}
\end{array}\right)=\left(\begin{array}{c}
\left\langle E_{x}, 1\right\rangle \\
\left\langle E_{x}, x\right\rangle \\
\left\langle E_{x}, x^{2}\right\rangle \\
\left\langle E_{x}, x^{3}\right\rangle
\end{array}\right) .
$$

The matrix in the above is known as the Gram matrix for the polynomial basis. The right-hand side represents the field projection onto the space of polynomials.

Now we need to select an appropriate inner product. By weighting the inner product, we can achieve a much more accurate expansion (in the average). To this aim, a good choice of weighting factor is the particle distribution itself. This weight has the effect of stretching the space in the regions of high particle density and nullifying the space outside the beam. Consequently, regions of high density contribute proportionally more in the determination of expansion coefficients while regions of space with no beam contribute nothing. This inner product is defined as

$$
\langle u, v\rangle \equiv \frac{1}{q} \iiint_{\mathfrak{R}^{3}} u(x, y, z) v(x, y, z) \rho(x, y, z) d x d y d z .
$$

Thus, we see that the Gram matrix in Eq. (58) is composed of the $x$ plane moments $\left\langle x^{m}\right\rangle$ while the right-hand side contains the field moments $\left\langle x^{m} E_{x}\right\rangle$.

We assume that the density function is given by Eq. (2), and the fields follow from Eq. (3). Then the nonzero coefficients have the values

$$
\begin{aligned}
& a_{1}=\frac{Q}{8 \pi \varepsilon_{0}} \Gamma_{1}(f) \frac{1}{X Y Z} \xi\left(\frac{X}{\sqrt{Y Z}}\right)+\frac{Q}{8 \pi \varepsilon_{0}} \Gamma_{1}^{\prime}(f) \frac{1}{Y^{3 / 2} Z^{3 / 2}} \xi\left(\frac{X}{\sqrt{Y Z}}\right) \\
& a_{3}=\frac{Q}{8 \pi \varepsilon_{0}} \Gamma_{3}(f) \frac{1}{X^{3} Y Z} \xi\left(\frac{X}{\sqrt{Y Z}}\right)+\frac{Q}{8 \pi \varepsilon_{0}} \Gamma_{3}^{\prime}(f) \frac{1}{X^{2} Y^{3 / 2} Z^{3 / 2}} \xi^{\prime}\left(\frac{X}{\sqrt{Y Z}}\right)
\end{aligned}
$$

where the $\Gamma_{i}$ and $\Gamma_{i}^{\prime}$ are functions of the distribution. To continue with this analysis, the next order of business is to expand the $a_{n}$ in the parameter $\varepsilon$, then apply the Fredholm alternative theorem to the resulting equations of motion. Here, it is obviously much easier to identify the effects of bunch core oscillations and the contributions of field nonlinearities.

CKAllen_ICFA_Workshop.doc submitted to World Scientific 02/10/00: 


\section{Acknowledgements}

The author thanks Tom Wangler for his support and guidance during this work.

\section{References}

[1] C.K. Allen, "Bunched Beam Envelope Equations Including Image Effects from a Cylindrical Pipe", Phys. Rev. E, Vol. 55, No. 6-B (1997), pp. 7591-7605.

[2] K. Bongardt, M. Pabst and A. Letchford, "Halo Formation by Mismatch for High Intensity Bunched Beams", Proceedings Linac Conf. 1999.

[3] B.C. Carlson, SIAM Journal on Mathematical Analysis, Vol. 8 (1977), pp. 231242.

[4] B.C. Carlson, Math. Comp., Vol. 49 (1987), pp. 595-606.

[5] See any introductory text on ordinary differential equations such as E.A. Coddington and N. Levinson, Theory of Ordinary Differential Equations (McGraw-Hill, New York, 1955).

[6] R.L. Gluckstern, A.V. Fedotov, S. Kurennoy and R. Ryne, "Halo Formation in Three-Dimensional Bunches", Phys. Rev. E, Vol. 58, No. 4 (1998), pp. $4977-$ 4990.

[7] R.L. Gluckstern, "Scalar Potential for Charge Distributions with Ellipsoidal Symmetry", Fermilab Internal Report TM-1402 (1986).

[8] M.H. Holmes, Introduction to Perturbation Methods (Springer-Verlag, New York, 1995) pp. 287-291.

[9] O.D. Kellogg, Foundations of Potential Theory (Dover, 1953) pp. 192-194.

[10] A.H. Nayfeh and D.T. Mook, Nonlinear Oscillations (Wiley, New York, 1979).

[11] M. Reiser and N. Brown, "Thermal Distributions of Relativistic Particle Beams with Space Charge", Phys. Rev. Lett., Vol. 71, No. 18, (1993), pp 2911-2914.

[12] F.R. Sacherer, "RMS Envelope Equations with Space Charge", IEEE Trans. Nucl. Sci., Vol. NS-18 (1971), pp. 1105-1107.

[13] I. Stakgold, Green's Functions and Boundary Value Problems (Wiley, New York, 1979), pp. 210-214.

[14] T.P. Wangler, K.R. Crandall, R. Ryne and T.S. Wang, "Particle-core model for transverse dynamics of beam halo", Phys. Rev. Special Topics, Accel. and Beams, Vol. 1, No. 084201 (1998).

[15] T.P. Wangler, "Beam Halo Formation in High Intensity Proton Beams", these proceedings.

[16] T.S. Wang, "Particle-core study of halo dynamics in periodic-focusing channels", Phys. Rev. E, Vol. 61, No. 1 (2000), pp. 855-861.

CKAllen_ICFA_Workshop.doc submitted to World Scientific 02/10/00: 